\title{
Surgical ablation of atrial fibrillation with a novel bipolar radiofrequency device
}

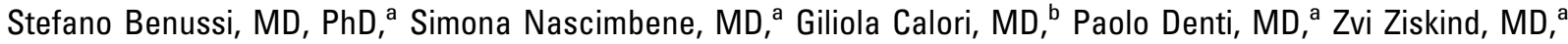 \\ Samer Kassem, MD, ${ }^{a}$ Giovanni La Canna, MD, ${ }^{a}$ Carlo Pappone, MD, PhD, ${ }^{c}$ and Ottavio Alfieri, MD ${ }^{a}$
}

Objective: When used for epicardial ablation, unipolar devices do not predictably yield transmural scars. Bipolar radiofrequency proved highly effective on the animal model, but clinical experience is still initial. We describe acute electrophysiologic findings and follow-up results of epicardial ablation with a novel bipolar radiofrequency device.

Methods: A bipolar ablator was used to perform a simplified left atrial lesion set in 90 consecutive patients with atrial fibrillation undergoing open heart surgery. Pacing thresholds were assessed during surgery to validate 24 pulmonary vein encircling lines (12 patients). Follow-up was $100 \%$ complete.

Results: In 67 of 90 patients (84\%), mitral valve disease was the main indication to surgery. Atrial fibrillation was continuous in 74 patients (82\%) and intermittent in 16 patients (18\%). Pacing threshold assessment showed a complete conduction block in 22 of 24 pulmonary vein couples (92\%) after a single ablation and in all patients after doubling of the encircling lines. No complications related to the ablation procedure were recorded. The sinus rhythm restoration rate was $79 \%$ at 3 months, $87 \%$ at 6 months, and $89 \%$ (17/18 patients) at 1 year. Postablation organized arrhythmias consisted in right atrial flutter in 2 patients $(2 \%)$ and left atrial flutter in 6 patients (7\%).

Conclusions: Epicardial ablation with bipolar radiofrequency grants acute transmurality. A simplified lesion set proved highly effective in eliminating atrial fibrillation at 1-year follow-up. Our data suggest that addition of a lesion to the mitral annulus is advisable to prevent left atrial flutter.

From the Division of Cardiac Surgery, ${ }^{\mathrm{a}}$ Epidemiology Unit, ${ }^{\mathrm{b}}$ and Division of Arrhythmology, ${ }^{c}$ S Raffaele University Hospital, Milan, Italy.

Received for publication Sept 29, 2004; revisions received Jan 10, 2005; accepted for publication Jan 13, 2005.

Address for reprints: Stefano Benussi, MD, $\mathrm{PhD}$, Division of Cardiac Surgery, S Raffaele University Hospital, via Olgettina 60, 20132 Milan, Italy (E-mail: stefano.benussi@ hsr.it).

J Thorac Cardiovasc Surg 2005;130:491-7

$0022-5223 / \$ 30.00$

Copyright $\odot 2005$ by The American Association for Thoracic Surgery

doi:10.1016/j.jtcvs.2005.01.009
$\mathrm{D}$ uring the past few years, diverse simplified surgical approaches, all derived from the original Cox maze procedure, have been devised to treat atrial fibrillation (AF). ${ }^{1-5}$ The principles inspiring such modifications have been the replacement of incisions with ablations and the reduction of the number of atrial lines. This has greatly increased accessibility and feasibility of surgery for AF. ${ }^{6}$

After the first reports at the turn of the millennium, ${ }^{1,7}$ epicardial ablation has been adopted by many groups for the concomitant treatment of $\mathrm{AF}^{8-10}$ and as an option for curing lone AF.,11

Diverse unipolar ablating systems have been used for epicardial ablation on the beating heart, with success rates between $77 \%$ and $90 \% .^{9,10,12}$ The major limitation of unipolar devices is that, because of the convective cooling (or heating) exerted by blood flowing through the atria and due to the composition of the diseased atrial wall, they do not predictably yield transmural lesions when used epicardially. ${ }^{9,13}$ Bipolar devices seem a promising way to obviate such problems: The first experimental trials demonstrated that bipolar radiofrequency (RF) ablation can reliably produce continuous transmural linear scars. ${ }^{14,15}$ Recent clinical reports document excellent early rhythm outcomes after bipolar ablation was used to perform a left atrial lesion $\operatorname{set}^{16}$ or a maze procedure. ${ }^{17,18}$ 
TABLE 1. Preoperative data

\begin{tabular}{lc}
\hline Patients & $90 s$ \\
\hline Male/female & $43 / 47$ \\
Age at surgery (y) & $64(55-69)$ \\
NYHA I (patients) & $7(8 \%)$ \\
NYHA II (patients) & $47(52 \%)$ \\
NYHA III (patients) & $35(39 \%)$ \\
NYHA IV (patients) & $1(1 \%)$ \\
Ejection fraction (\%) & $60(54-60)$ \\
Ejection fraction $\leq 40 \%$ (patients) & $11(12 \%)$ \\
Left atrial diameter (mm) & $54(49-59)$ \\
Intermittent AF (patients) & $16(18 \%)$ \\
Continuous AF (patients) & $74(82 \%)$ \\
Duration of AF (mo) & $15(9-49)$ \\
Permanent pacemaker (patients) & $2(2 \%)$ \\
Systolic pulmonary artery pressure (mm Hg) & $48(40-55)$ \\
Left ventricular telediastolic pressure $(\mathrm{mm})$ & $53(49-60)$ \\
Left ventricular telediastolic volume (mL) & $101(76-137)$
\end{tabular}

NYHA, New York Heart Association functional class; $A F$, atrial fibrillation. Values are expressed as number and (percentage) for discreet variables and as number (25th, 75th percentiles) for continuous variables.

We report our ablation technique with a novel bipolar RF system, describing early electrophysiologic findings and follow-up results.

\section{Methods}

Concomitant AF treatment with bipolar RF was performed in 90 patients between February 2003 and March 2004. Indications for the ablation procedure were continuous AF (lasting at least 6 months) in 74 patients (82\%) and symptomatic intermittent $\mathrm{AF}$ refractory to antiarrhythmic medications or to prior percutaneous ablation in 16 patients (18\%). Seventy-six patients $(84 \%)$ had primary mitral valve disease as the main indication to surgery. Preoperative data are summarized in Table 1.

Informed written consent was signed by each patient before surgery.

To decrease the risk of intracavitary thrombus formation, a continuous heparin infusion was administered until 6 hours before surgery. A preoperative transesophageal echocardiography was performed in all patients no longer than 24 hours before surgery to exclude left atrial thrombosis.

All ablations were performed using a novel bipolar RF ablation system (Cobra Bipolar, Boston Scientific, San Jose, Calif). Two 64-mm-long ablating inserts are mounted on the opposing faces of the jaws of a stainless steel clamp. Each insert is made of $2 \mathrm{RF}$ electrodes embedded in a polyester covering. The electrodes have a thermocouple mounted on each end. Different shapes of the clamp can be chosen on the basis of the ablation site and the surgeon's preference. RF current was delivered for 40 to 45 seconds at 35 to $40 \mathrm{~W}$, with a preset temperature of $90^{\circ} \mathrm{C}$.

All the ablations were performed on full cardiopulmonary bypass at $37^{\circ} \mathrm{C}$.

The pattern was basically the same as we described earli$\mathrm{er}^{3,12}$; the only exception was the mitral line, which we decided to omit when using bipolar RF because of concern about injur-

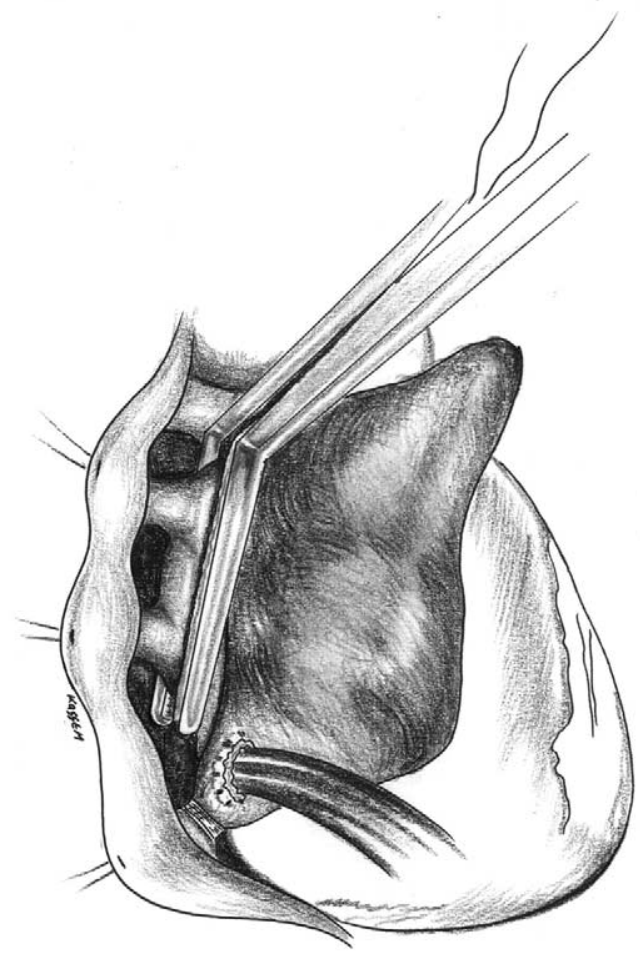

Figure 1. Encircling ablation with the bipolar radiofrequency (RF) clamp around the ostia of the right pulmonary veins.

ing a major coronary artery branch in the atrioventricular (A-V) groove.

After dissection of pericardial reflections, the bipolar device was clamped around the atrial cuff containing the inflow of the right pulmonary veins (Figure 1). RF energy was deployed yielding an encircling lesion. The heart was lifted, the Marshall fold was interrupted, and a similar encircling lesion was performed on the left pulmonary vein cuff (Figure 2). When intraoperative conduction block validation was not performed, the pulmonary vein lesions were always doubled. The appendage was ablated at its base, away from the A-V groove to not harm a major coronary artery, and connected to the left encircling by means of an additional ablation with the tip of the clamp pinching the left encircling line and the base ending in the body of the left appendage. The line connecting the 2 encirclings was usually performed through a left atriotomy (Figure 3). One jaw of the clamp was inserted in the left atrium with the tip in the upper left pulmonary vein or in the appendage, while the other jaw was outside, in the transverse sinus of pericardium. Figure 3 depicts the final set of lines. After the ablations, the appendage was sutured, generally from the inside.

\section{Postoperative Management and Follow-up}

Amiodarone was the first-choice antiarrhythmic drug for postoperative prophylaxis. In the presence of contraindications to amiodarone, sotalol or other medications were given as appropriate. 


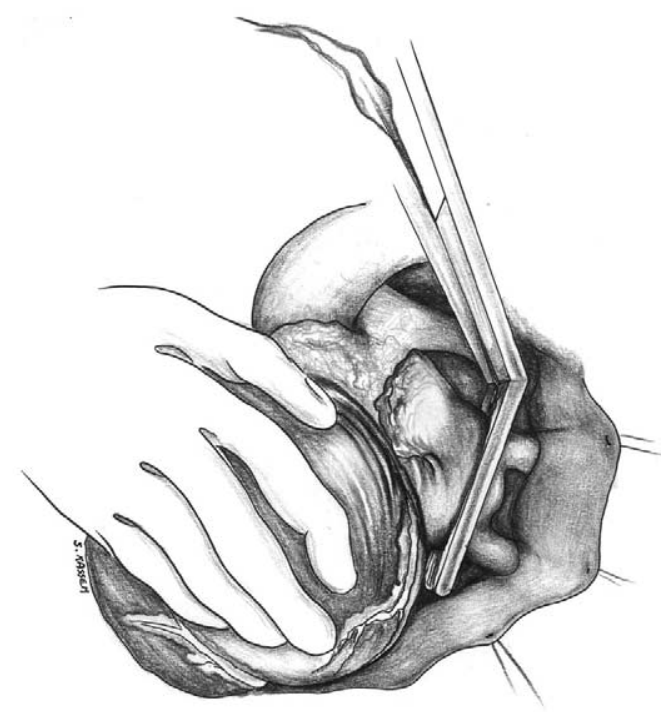

Figure 2. Epicardial beating heart ablation around the left pulmonary veins ostia.

Antiarrhythmic prophylaxis was generally continued for 3 to 6 months.

Standard 12-lead electrocardiograph, Holter, and transthoracic echocardiography monitoring were performed $1,3,6$, and 12 months after the operation.

Recurrences of the arrhythmia were treated by improving the medical treatment. Patients with AF despite optimal medical therapy had at least 1 attempt of external direct-current (DC) shock.

Six months after surgery, oral anticoagulants were discontinued in patients with stable sinus rhythm and bilateral atrial contraction, in the absence of any other indications.

\section{Conduction Block Validation}

Electrical isolation of right and left pulmonary vein couples was acutely assessed in 12 patients. Two monopolar electrodes were attached to the intrapericardial portion of the pulmonary vein couple (1 electrode on each vein), and DC shock was administered when needed. The pacing threshold was then assessed before and after the encircling lesion, on cardiopulmonary bypass.

\section{Statistical Analysis}

The following variables were analyzed for a possible correlation with the occurrence of AF, left atrial flutter (defined as any form of atypical flutter with isoelectric or negative $\mathrm{P}$ waves in aVL and positive $\mathrm{P}$ waves in $\mathrm{V} 1$ and $\mathrm{III}$ ), and any type of left atrial arrhythmia (AF or left flutter) at follow-up. Preoperative variables included age, gender, left atrial diameter, type (continuous vs intermittent) and duration of AF, ejection fraction, systolic pulmonary artery pressure, left ventricular telediastolic pressure and volume, New York Heart Association functional class, type of associated organic heart disease, cause of mitral disease (degenerative vs rheumatic), and prior heart surgery. Operative variables included type of surgical procedure, reconstructive versus replacement valve surgery, and duration of cardiopulmonary bypass and cardiac arrest. Postoperative variables included total bleeding, reexploration, type of antiarrhythmic prophylaxis, overall intensive care unit, and hospital stay. Such variables, as assessed by the Kolmogorov-Smirnov test, were non-normally distributed. Therefore, values are expressed as median, 25th and 75th percentiles $\left(\mathrm{Q}_{1} ; \mathrm{Q}_{3}\right)$ for continuous variables, and as $\mathrm{N}(\%)$ for categoric variables, unless stated otherwise.

The possible association between arrhythmia recurrence at each time point and all other variables was investigated by chi-square test and Mann-Whitney $U$ test as appropriate. Multiple logistic regression analysis was run including all variables that resulted in $P$ values less than .1 at univariate analysis. To account for multiple
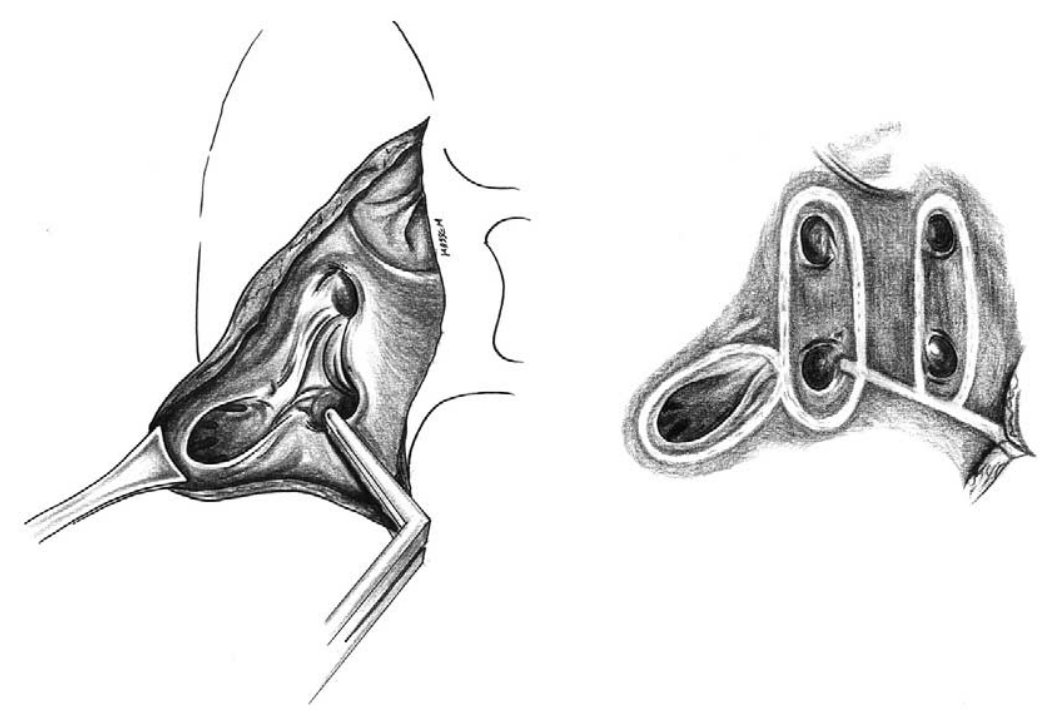

Figure 3. Connecting lesion from the atriotomy to the left upper pulmonary vein (left) and final set of ablation lines (right). 
TABLE 2. Surgical procedures

\begin{tabular}{lc}
\hline Patients & $\mathbf{9 0}$ \\
\hline Mitral repair & $36(40 \%)$ \\
Anuloplasty & $10(11 \%)$ \\
Edge to edge & $15(17 \%)$ \\
Quadrangular resection & $11(12 \%)$ \\
Mitral commissurotomy & $4(4 \%)$ \\
Mitral replacement & $40(44 \%)$ \\
Biologic & $8(9 \%)$ \\
Mechanical & $32(36 \%)$ \\
Tricuspid annuloplasty & $44(49 \%)$ \\
CABG & $5(6 \%)$ \\
Aortic valve replacement & $13(14 \%)$ \\
Biologic & $5(6 \%)$ \\
Mechanical & $8(9 \%)$ \\
VSD repair & $1(1 \%)$ \\
ASD repair & $6(7 \%)$ \\
Replacement of ascending aorta & $2(2 \%)$ \\
Two or more procedures & $56(62 \%)$ \\
\hline
\end{tabular}

$C A B G$, Coronary artery bypass grafting; $V S D$, ventricular septal defect; $A S D$, atrial septal defect.

tests at each time point, the Bonferroni correction was applied and the $P$ value was set at .01 .

\section{Results}

\section{Operative Results, Morbidity, and Mortality}

The open heart procedures are detailed in Table 2. Cardiopulmonary bypass time was 93 minutes (81-106 minutes). Aortic crossclamp duration was 63 minutes (53-78 minutes). The time spent ablating during aortic occlusion never exceeded 2 minutes.

No complication related to bipolar ablation was recorded. Four patients (4\%) required reexploration for bleeding. Overall, intensive care unit stay was 2 days (1-4 days) and postoperative hospital stay was 7 days (6-12 days). Major complications occurred in 2 patients. A 65-year-old female patient had a type $\mathrm{B}$ aortic dissection with transient lower limb ischemia, acute renal failure, and respiratory insufficiency 12 hours after mitral and tricuspid repair, plication of the ascending aorta, and ablation. Another 65year-old woman underwent emergency percutaneous transluminal coronary angioplasty immediately after arrival in the intensive care unit because of acute embolic occlusion of the circumflex artery after mitral valve replacement, tricuspid repair, and ablation. One 79-year-old woman died 37 days after mitral valve replacement, tricuspid repair, and ablation because of rectus muscles hematoma leading to acute renal failure and eventually to respiratory insufficiency.

Follow-up was $100 \%$ complete (median 7 months; 4-9 months).

No thromboembolic event was recorded, and no patient died.

\section{Conduction Block Validation}

The baseline pacing threshold on 24 pulmonary vein pairs was $3.3 \mathrm{~mA}$ (2.4-4 mA). After a single RF bipolar ablation on cardiopulmonary bypass, pacing showed a complete conduction block (no pacing at $\geq 20 \mathrm{~mA}$ ) in 22 of 24 pulmonary vein couples $(92 \%)$. In 2 right pulmonary vein couples, the pacing threshold remained unchanged after ablation (5 $\mathrm{mA}$ in 1 patient and $2 \mathrm{~mA}$ in 1 patient). A repeat application led to complete block in both cases.

\section{Rhythm}

All patients were free from $\mathrm{AF}$ at arrival in the intensive care unit. Thirty-nine patients (43\%) experienced some form of sustained supraventricular arrhythmia during the hospital stay. At discharge, 53 of 89 patients $(60 \%$; $95 \%$ confidence interval [CI]: 49\%-70\%) were in normal sinus rhythm. The proportion of patients with a recovered sinus rhythm was then $79 \%$ (95\% CI: $68 \%-87 \%)$ at 3 months, $87 \%$ (95\% CI: $75 \%-95 \%)$ at 6 months, and $89 \%$ (95\% CI: 65\%-99\%) at 1-year control (Figure 4).

Overall, 38 patients (43\%) underwent at least 1 DC shock at any time after surgery. Eight patients (9\%) had 2 or more attempts.

The occurrence of AF or left flutter at 3 months was influenced by preoperative left atrial diameter $(P=.0014)$. At multivariate analysis the odds ratio for each $0.5-\mathrm{cm}$ increment of left atrial diameter was 1.49 (95\% CI: $1.23 \%$ $1.81 \%)$. No other significant correlation was found. In particular, no predictor of left flutter was identified.

A permanent pacemaker was implanted in 1 patient with preoperative sick sinus syndrome.

Two patients $(2 \%)$ experienced typical counterclockwise right atrial flutter 3 months after surgery and were successfully treated with percutaneous ablation of the cavo-tricuspid isthmus. Six patients $(7 \%)$ had left atrial flutter, all of them within 3 months from mitral surgery. One patient recovered sinus rhythm with antiarrhythmic medications, and 1 patient required DC shock and medications. Two patients underwent an electrophysiologic study with a transseptal approach at 3 months. Both studies showed an optimal isolation of the pulmonary veins. In both cases a reentry circuit around the mitral valve annulus was documented and interrupted through a left isthmus RF ablation line. In 1 patient a reentry circuit around a right atriotomy incision also required ablation. Both patients recovered sinus rhythm at the completion of the procedure, without electrical cardioversion.

Two patients are presently waiting to be admitted for electrophysiologic assessment.

Of 24 patients followed up for more than 6 months, 12 (50\%) were not receiving drugs, 3 (12.5\%) were receiving low-dose sotalol, and 3 (12.5\%) were receiving $\beta$-blockers. 


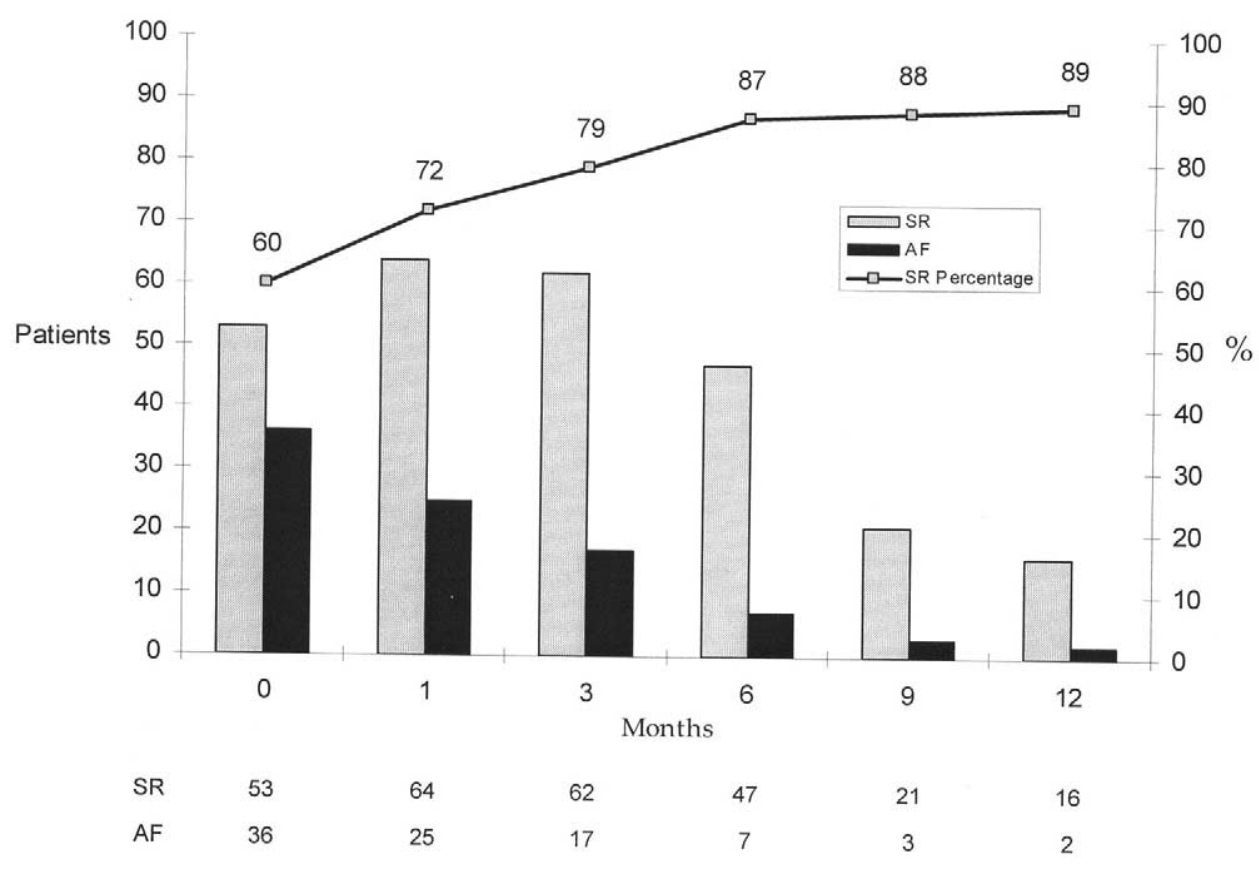

Figure 4. Rhythm after surgery. $S R$, Sinus rhythm; $A F$, atrial fibrillation.

Only 6 patients $(25 \%)$ were still receiving low-dose amiodarone.

All transthoracic echo-Doppler controls performed 3 or more months after surgery in patients in sinus rhythm showed an effective contraction (A-wave $>10 \mathrm{~cm} / \mathrm{s}$ ) of both atria.

\section{Discussion}

The rate of sinus rhythm maintenance after concomitant surgical treatment of AF through bipolar RF left atrial ablation improves progressively during the first year after surgery. At 1 year, $89 \%$ of the patients were free from AF in our series. Our data are in line with the good early clinical results recently reported by other groups studying intraoperative bipolar RF ablation. ${ }^{16-18}$ Of note, such results compare favorably with the $60 \%$ to $92 \%$ success rate reported at 6 to 12 months after left atrial ablation with the diverse unipolar devices available for clinical use. 1,2,12,19 $^{2}$

Success rates in excess of $95 \%$ have only been described after the maze operation. ${ }^{20,21}$ But such technique has never become popular in the context of concomitant AF ablation, because it is technically demanding and it significantly increases postoperative morbidity. ${ }^{22}$ Moreover, in experienced hands, a concomitant maze carries a $20 \%$ or greater risk of postoperative pacemaker implantation. ${ }^{21}$ Some of the lines of the original maze procedure are today deemed redundant. Cox has recently proposed performing only the left part of the maze and a cavo-tricuspid ablation line. ${ }^{6}$

In an earlier series of patients treated with unipolar RF, in whom the same lesion set was completed by a mitral line, our 1-year success rate was $79 \% .^{12}$ So, despite a simplification of the lesion set, in our hands the efficacy of bipolar RF seems, if anything, slightly superior to that of unipolar RF.

Data on transmurality of beating-heart epicardial ablations with unipolar devices are all but satisfactory. Although acutely effective in most cases, epicardial microwave ablation on the beating heart at times fails to accomplish electrical isolation even after repeat ablation. ${ }^{9}$ This is true also for unipolar RF ablation, and it has been related by Santiago et $\mathrm{al}^{13}$ to the composition and thickness of the atrial wall. The major obstacle to the progression of the lesion through the subendocardial layer is the convective cooling exerted by blood flow through the atrial chamber.

Bipolar systems are specifically designed to work epicardially. The clamping mechanism abates the endocardial convective cooling and reduces tissue thickness by compression, at the same time improving contact. Prasad and colleagues $^{14}$ demonstrated atrial ablations achieved with bipolar RF to be predictably transmural both acutely and after 3 months in pigs. In our series pacing threshold assessment showed a complete conduction block in $92 \%$ $(22 / 24)$ of the pulmonary vein couples after a single ablation and in $100 \%$ after 1 repeat ablation. These data are similar to those described by Gillinov and McCarthy ${ }^{23}$ with a similar device and confirm the efficacy of bipolar RF. Interestingly, the 2 ineffective encircling ablations left the pacing threshold completely unchanged. This all-or-nothing effect suggests a gap caused by suboptimal positioning of the clamp (incomplete clamping or local folding of the atrial 
wall leading to double thickness) rather than incomplete transmurality of the lesions.

On the basis of our experience, it is safe to assume that in the absence of any validation system, epicardial isolation of the pulmonary veins on cardiopulmonary bypass can be predictably achieved with bipolar RF by performing 2 parallel ablations. This generally takes less than 2 minutes for each pulmonary vein pair after minor dissection of the posterior pericardial reflections.

Another important aspect of bipolar ablation is the safety profile. Injury to the esophagus or to the bronchial tree has been found to occur in as much as $1 \%$ of patients ablated with unipolar RF. ${ }^{24}$ Collateral damage of the structures surrounding the heart has not occurred in this series, and it is actually very unlikely with bipolar ablation. In fact, during the ablation, only the outer, inactive part of the clamp is in contact with the parietal pericardium. Only the tissue that is held between the jaws is heated significantly, and thermal spread is thus minimized.

The increased swiftness and safety of the ablation procedure stimulated in our experience an expansion of the indications in patients with a poor ventricular function and in those requiring complex and/or multiple open heart procedures.

As already reported after other ablation procedures, ${ }^{1,5,7,19}$ the high rate of early supraventricular arrhythmias (43\% in this series) justifies the routine use of antiarrhythmic prophylaxis and a more liberal use of DC shock during the first postoperative weeks. From 3 to 6 months after surgery, the rhythm stabilizes, rendering withdrawal of amiodarone possible in most patients.

A larger left atrial diameter at 3-month follow-up was a predictor of the occurrence of relapsing AF or left atrial flutter. One possible explanation is that a simplified left atrial approach as the one described is less effective in curing those "worse" cases with a larger atrium. But still, such correlation was not a constant finding, and it needs to be confirmed by larger series and longer follow-up. In fact, the smaller sample size at latest controls might have prevented some predictors to be significant in the present study.

The only patient requiring implantation of a permanent pacemaker after surgery had a bradytachycardia syndrome before surgery. This confirms our and others' impression that the avoidance of any right atrial ablation line renders postoperative sinus node dysfunction a rare occurrence. ${ }^{1,5,8,9,12,19}$ On the other hand, a cavo-tricuspid line would most probably have prevented the occurrence of typical right atrial flutter, which required percutaneous ablation in 2 of our patients.

More worrisome is the $7 \%$ rate of postoperative left atrial flutter registered within the first 3 months in this series. From a clinical standpoint, left atrial flutter is in fact probably the worst postablation arrhythmia because it is not easily managed by drugs, more often than not it relapses after DC shock, and it requires complex transseptal mapping and ablation procedures. It is noteworthy that in our prior experience with unipolar RF the incidence of left flutter/ tachycardia was less than $1 \%$. A possible explanation is that the omission of the mitral line, because of the potential risk of coronary injury during bipolar ablation in this area, has actually left the substrate for a left isthmus reentry unaddressed. This hypothesis is substantiated by the finding of a reentry circuit around the mitral valve annulus in both patients with left atrial flutter undergoing electrophysiologic study in this series.

The mitral line has been a cornerstone in AF surgery ever since the original design of the maze operation ${ }^{25,26}$ and is now also widely applied by electrophysiologists. ${ }^{27,28} \mathrm{An}$ incompleteness of the mitral line has been related to some cases of failure of the ablation procedure. ${ }^{12,29}$

Despite the good sinus rhythm recovery rates we recorded, such a substantial incidence of postoperative atypical flutter deserves consideration. While virtually allowing the completion of the ablation procedure epicardially on the beating heart, the omission of the mitral line may increase the likelihood of left flutter, turning a bad arrhythmia into a worse one. Even if such arrhythmia can be dealt with in the electrophysiology laboratory, at present this requires considerable expertise with transseptal procedures. Therefore, especially if a specific electrophysiologic expertise is not available, according to our findings, omitting the mitral line may not be the most sensible option when using bipolar RF.

Other alternative strategies should then be taken into consideration such as the following:

- Complete the mitral line with a different unipolar device (eg, a reusable cryoprobe when this is available) in the coronary-free segment of the A-V groove. ${ }^{30}$

- Perform the ablation with bipolar RF or a unipolar ablator after surgical dissection of the $\mathrm{A}-\mathrm{V}$ junction to isolate the coronary artery.

Unfortunately, among the available ablation systems, no catheter is presently usable in both the bipolar and the unipolar modes.

\section{Conclusions}

Bipolar RF ablation of the left atrium grants acute electrical isolation. A simplified left atrial lesion set performed with bipolar RF is highly effective in eliminating AF at 1 year after open surgery. The safety profile and negligible addition of time required make bipolar RF a suitable option for the concomitant treatment of AF even in the most complex cases and in those with a poorer left ventricular function.

Our experience suggests that the addition of a lesion to the mitral annulus is advisable to prevent left atrial flutter. 


\section{References}

1. Sueda T, Nagata H, Shikata H, Orihashi K, Morita S, Sueshiro M, et al. Simple left atrial procedure for chronic atrial fibrillation associated with mitral valve disease. Ann Thorac Surg. 1996;62:1796-800

2. Melo JQ, Neves J, Adragao P, Ribeiras R, Ferreira MM, Bruges L, et al. When and how to report results of surgery on atrial fibrillation. Eur J Cardiothorac Surg. 1997;12:739-44.

3. Benussi S, Pappone C, Nascimbene S, Oreto G, Caldarola A, Stefano PL, et al. A simple way to treat atrial fibrillation during mitral valve surgery: the epicardial radiofrequency approach. Eur J Cardiothorac Surg. 2000;17:524-9.

4. Sie HT, Beukema WP, Misier AR, Elvan A, Ennema JJ, Haalebos $\mathrm{MM}$, et al. Radiofrequency modified maze in patients with atrial fibrillation undergoing concomitant cardiac surgery. J Thorac Cardiovasc Surg. 2001;122:249-56.

5. Williams MR, Stewart JR, Bolling SF, Freeman S, Anderson JT, Argenziano M, et al. Surgical treatment of atrial fibrillation using radiofrequency energy. Ann Thorac Surg. 2001;71:1939-44.

6. Cox JL. Atrial fibrillation II: rationale for surgical treatment. J Thorac Cardiovasc Surg. 2003;126:1693-9.

7. Benussi S, Pappone C, Nascimbene S, Oreto G, Blasio A, Caldarola A, et al. Epicardial radiofrequency ablation of chronic atrial fibrillation during mitral valve surgery: early success rate and atrial function recovery. Circulation. 1999;100:I854.

8. Melo J, Adragao P, Neves J, Ferreira M, Timoteo A, Santiago T, et al. Endocardial and epicardial radiofrequency ablation in the treatment of atrial fibrillation with a new intra-operative device. Eur J Cardiothorac Surg. 2000;18:182-6.

9. Maessen JG, Nijs JF, Smeets JL, Vainer J, Mochtar B. Beating-heart surgical treatment of atrial fibrillation with microwave ablation. Ann Thorac Surg. 2002;74:1307-11.

10. Raman JS, Seevanayagam S, Storer M, Power JM. Combined endocardial and epicardial radiofrequency ablation of right and left atria in the treatment of atrial fibrillation. Ann Thorac Surg. 2001;72:1096-9.

11. Saltman AE, Rosenthal LS, Francalancia NA, Lahey SJ. A completely endoscopic approach to microwave ablation for atrial fibrillation. Heart Surg Forum. 2003;6:38-41.

12. Benussi S, Nascimbene S, Agricola E, Calori G, Calvi S, Caldarola A, et al. Surgical ablation of atrial fibrillation using the epicardial radiofrequency approach: mid-term results and risk analysis. Ann Thorac Surg. 2002;74:1050-6.

13. Santiago T, Melo J, Gouveia RH, Neves J, Abecasis M, Adragao P, et al. Epicardial radiofrequency applications: in vitro and in vivo studies on human atrial myocardium. Eur J Cardiothorac Surg. 2003; 24:481-6.

14. Prasad SM, Maniar HS, Diodato MD, Schuessler RB, Damiano RJ Jr. Physiological consequences of bipolar radiofrequency energy on the atria and pulmonary veins: a chronic animal study. Ann Thorac Surg. 2003;76:836-41.

15. Bonanomi G, Schwartzman D, Francischelli D, Hebsgaard K, Zenati MA. A new device for beating heart bipolar radiofrequency atrial ablation. J Thorac Cardiovasc Surg. 2003;126:1859-66.
16. Gillinov AM, McCarthy PM. Advances in the surgical treatment of atrial fibrillation. Cardiol Clin. 2004;22:147-57.

17. Gaynor SL, Diodato MD, Prasad SM, Ishii Y, Shuessler RB, Bailey MS, et al. A prospective, single-center clinical trial of a modified Cox maze procedure with bipolar radiofrequency ablation. J Thorac Cardiovasc Surg. 2004;128:535-41.

18. Mokadam NA, McCarthy PM, Gillinov AM, Ryan WH, Moon MR, Mack MJ, et al. A prospective, multicenter trial of bipolar radiofrequency ablation for atrial fibrillation: early results. Ann Thorac Surg. 2004;78:1665-70.

19. Pasic M, Bergs P, Muller P, Hofmann M, Grauhan O, Kuppe H, et al. Intraoperative radiofrequency maze ablation for atrial fibrillation: the Berlin modification. Ann Thorac Surg. 2001;72:1484-90.

20. Cox JL, Jaquiss RDB, Schuessler RB, Boineau JP. Modification of the maze procedure for the treatment of atrial flutter and fibrillation. I. Rationale and surgical results. J Thorac Cardiovasc Surg. 1995;110: 473-84.

21. Prasad SM, Maniar HS, Camillo CJ, Schuessler RB, Boineau JP, Sundt TM 3rd, et al. The Cox maze III procedure for atrial fibrillation: long-term efficacy in patients undergoing lone versus concomitant procedures. J Thorac Cardiovasc Surg. 2003;126:1822-8.

22. Kawaguchi AT, Kosakai Y, Sasako Y, Eishi K, Kiyoharu N, Kawashima Y. Risks and benefits of combined maze procedure for atrial fibrillation associated with organic heart disease. J Am Coll Cardiol. 1996;28:985-90

23. Gillinov AM, McCarthy PM. Atricure bipolar radiofrequency clamp for intraoperative ablation of atrial fibrillation. Ann Thorac Surg. 2002;74:2165-8

24. Doll N, Borger MA, Fabricius A, Stephan S, Gummert J, Mohr FW, et al. Esophageal perforation during left atrial radiofrequency ablation: is the risk too high? J Thorac Cardiovasc Surg. 2003;125:836-42.

25. Cox JL, Shuessler RB, D'Agostino HJ, Stone CM, Chang BC, Cain ME, et al. The surgical treatment of atrial fibrillation III. Development of a definitive surgical treatment. J Thorac Cardiovasc Surg. 1991; 101:569-83.

26. Cox JL. Atrial fibrillation I: a new classification system. J Thorac Cardiovasc Surg. 2003;126:1686-92.

27. Pappone C, Santinelli V, Manguso F, Vicedomini G, Gugliotta F, Augello G, et al. Pulmonary vein denervation enhances long-term benefit after circumferential ablation for paroxysmal atrial fibrillation. Circulation. 2004;109:327-34.

28. Jais P, Weerasooriya R, Shah DC, Hocini M, Macle L, Choi KJ, et al. Ablation therapy for atrial fibrillation: past, present and future. Cardiovasc Res. 2002;54:337-46.

29. Cox JL, Ad N. The importance of cryoablation of the coronary sinus during the Maze procedure. Semin Thorac Cardiovasc Surg. 2000;12: 20-4.

30. Benussi S, Nascimbene S, Calvi S, Alfieri O. A tailored anatomical approach to prevent complications during left atrial ablation. Ann Thorac Surg. 2003;75:1979-81. 